\title{
Genetic Variants in Toll-Like Receptor 4 Gene and Their Association Analysis with Estimated Glomerular Filtration Rate in Mexican American Families
}

\author{
Farook Thameem ${ }^{a}$ e Sobha Puppala ${ }^{b}$ Vidya S. Farook ${ }^{d}$ \\ Balakuntalam S. Kasinath $^{a, c}$ John Blangero ${ }^{d}$ Ravindranath Duggiralad \\ Hanna E. Abboud ${ }^{a, c}$ \\ ${ }^{a}$ Division of Nephrology, Department of Medicine, University of Texas Health Science \\ Center, ${ }^{b}$ Department of Genetics, Texas Biomedical Research Institute, and 'South Texas \\ Veterans Healthcare System, San Antonio, Tex., and d South Texas Diabetes and Obesity \\ Institute, University of Texas Rio Grande Valley, Brownsville, Tex., and Edinburg, Tex., USA; \\ e Department of Biochemistry, Faculty of Medicine, Kuwait University, Kuwait City, Kuwait
}

\section{Key Words}

Toll-like receptor 4 · Asp(299)Gly · Thr(399)Ile · rs4986790 · rs4986791 · Estimated glomerular filtration rate $\cdot$ Mexican Americans

\begin{abstract}
Background/Aim: Toll-like receptor 4 (TLR4) is one of the regulators of the innate immune response. Genetic variations in TLR 4 have been associated with inflammatory diseases, including type 2 diabetes. However, to our knowledge, there are no reports on the role of variations in TLR4 in chronic kidney disease susceptibility. The objective of this study is to determine whether the genetic variants in TLR4 are associated with the estimated glomerular filtration rate (eGFR), a measure of renal function. Methods: To evaluate the association between TLR4 variants and eGFR, we used data obtained from 434 Mexican American participants from the San Antonio Family Diabetes/Gallbladder Study. GFR was estimated using the Modification of Diet in Renal Disease formula. The Asp(299)Gly (rs4986790) and Thr(399)Ile (rs4986791) variants of TLR4 were genotyped using the TaqMan assay. Association analyses between genotypes and eGFR were performed using the measured genotype approach. Results: Of the two genetic markers examined for association, only the Asp(299)Gly variant of TLR4 exhibited a nominally significant association with eGFR $(p=0.025)$ after accounting for the covariate effects of age and sex terms, diabetes, duration of diabetes, systolic blood pressure, body mass
\end{abstract}


Thameem et al.: Genetic Variants in Toll-Like Receptor 4 Gene and Their Association

Analysis with Estimated Glomerular Filtration Rate in Mexican American Families

index, and antihypertensive treatment. Carriers of Gly299 had significantly decreased eGFR values. Although, the Thr(399)Ile variant failed to exhibit a statistically significant association with eGFR, the carriers of Ile399, however, showed a trend towards decrease in eGFR. Conclusion: We show for the first time that Asp(299)Gly variants of TLR4 are associated with decrease in renal function in Mexican Americans.

(C) 2016 S. Karger AG, Base

\section{Introduction}

Chronic kidney disease (CKD), defined as reduction in estimated glomerular filtration rate (eGFR), affects about $15 \%$ of the US population. The incidence and prevalence rates of CKD are increasing worldwide, mirroring a substantial global disease burden, given that the risk for end-stage renal disease, cardiovascular morbidity, and overall mortality increases with declining GFR [1]. While CKD susceptibility is known to be influenced by traditional risk factors, such as age, hypertension, diabetes, and dyslipidemia, emerging evidence indicates that nontraditional risk factors, such as chronic inflammation-mediated activation of the innate immune system, also contribute to the pathogenesis of CKD and diabetic nephropathy (DN) $[2,3]$.

Toll-like receptor 4 (TLR4) is one of the key components of the mammalian innate immune system. Binding of exogenous and endogenous ligands activates TLR4, which in turn triggers a signaling cascade, leading to initiation of an adaptive and innate immune response. Genetic variations in TLR4 are thought to alter the ligand-binding capacity and contribute to the chronic inflammation-mediated pathogenesis of many diseases [4]. Particularly, the Asp(299)Gly and Thr(399)Ile variants that are located in the extracellular domain of TLR4 have been extensively examined for their association with inflammatory diseases $[5,6]$. The Asp(299)Gly and Thr(399)Ile variants of TLR4 have been demonstrated to cause conformational changes in the vicinity of the mutation area and affect its folding efficiency, cell surface expression, protein stability, as well as interaction with downstream signaling proteins [7]. These mutations also cause a 2-fold reduction in the expression level of TLR4 [8]. This reduction is further increased to 10 -fold in the absence of myeloid differentiation factor 2 that forms a complex with TLR4 and lipopolysaccharide [8, 9]. Furthermore, it has been shown that the Asp(299)Gly variant in TLR4 interferes with TLR4 interaction with the myeloid differentiation primary response 88 and other downstream signaling proteins [10].

To our knowledge, there are no reports on the frequencies of these two polymorphisms in the Mexican American population and their association with eGFR, a measure of renal function. Therefore, the aim of the present study is to assess whether the Asp(299)Gly and Thr(399)Ile variants in TLR4 are associated with eGFR in Mexican Americans, a minority population in the US at high risk for type 2 diabetes (T2DM) and its complications, including nephropathy.

\section{Materials and Methods}

The recruitment of Mexican American family members and data collection procedures of the San Antonio Family Diabetes/Gallbladder Study (SAFDGS) were reported previously [11]. In brief, probands were low-income Mexican Americans with T2DM, and all first, second, and third degree relatives of probands were invited to participate in the study. A variety of anthropometric, demographic, metabolic, and hemodynamic variables were collected from the participants. Blood samples were obtained after a 12-hour fast and collected again $2 \mathrm{~h}$ after a standard oral glucose load of $75 \mathrm{~g}$ for the assessment of plasma glucose levels. Diabetes was defined as fasting glucose levels $\geq 126 \mathrm{mg} / \mathrm{dl}$ and/or 2 -hour glucose levels $\geq 200 \mathrm{mg} / \mathrm{dl}$. Partic- 


\section{CardioRenal Medicine}

Table 1. Clinical characteristics of the SAFDGS participants with genotypic and phenotypic data used for this study $(n=434)$

\begin{tabular}{l|l}
\hline \multicolumn{2}{l}{ Cardiorenal Med 2016;6:301-306 } \\
\hline DOI: 10.1159/000445754 & $\begin{array}{l}\text { ( 2)16 S. Karger AG, Basel } \\
\text { www.karger.com/crm }\end{array}$ \\
\hline
\end{tabular}

Thameem et al.: Genetic Variants in Toll-Like Receptor 4 Gene and Their Association Analysis with Estimated Glomerular Filtration Rate in Mexican American Families

\begin{tabular}{lc}
\hline Phenotypes & Mean \pm SD or \% \\
\hline Age, years & $46.9 \pm 15.9$ \\
Female gender & 61 \\
T2DM & 29 \\
Duration of diabetes, years & $7.9 \pm 9.4$ \\
Hypertension & 34 \\
Systolic blood pressure, $\mathrm{mm} \mathrm{Hg}$ & $128.5 \pm 18.4$ \\
Diastolic blood pressure, $\mathrm{mm} \mathrm{Hg}$ & $71.3 \pm 9.7$ \\
Body mass index & $31.3 \pm 7.1$ \\
GFR-MDRD, $\mathrm{ml} / \mathrm{min} / 1.73 \mathrm{~m}^{2}$ & $89.0 \pm 18.9$ \\
\hline
\end{tabular}

Table 2. Model-derived mean values of eGFR by the genotype class of TLR4 variants

\begin{tabular}{|c|c|c|c|c|c|c|c|c|}
\hline \multirow[t]{3}{*}{ Trait } & \multicolumn{4}{|c|}{ rs4986790 (GAT to GGT) ${ }^{a}$} & \multicolumn{4}{|c|}{ rs4986791 (AGC to AAC) ${ }^{b}$} \\
\hline & \multicolumn{4}{|l|}{ Asp(299)Gly } & \multicolumn{4}{|l|}{ Thr(399)Ile } \\
\hline & $\begin{array}{l}\text { Asp/Asp } \\
(n=400)\end{array}$ & $\begin{array}{l}\text { Asp/Gly } \\
(\mathrm{n}=30)\end{array}$ & $\begin{array}{l}\text { Gly/Gly } \\
(\mathrm{n}=1)\end{array}$ & $\begin{array}{l}\mathrm{p} \\
\text { value }\end{array}$ & $\begin{array}{l}\text { Thr/Thr } \\
(n=403)\end{array}$ & $\begin{array}{l}\text { Thr/Ile } \\
(\mathrm{n}=30)\end{array}$ & $\begin{array}{l}\text { Ile/Ile } \\
(n=1)\end{array}$ & $\begin{array}{l}\mathrm{p} \\
\text { value }\end{array}$ \\
\hline $\begin{array}{l}\text { GFR-MDRD } \\
\left(\mathrm{ml} / \mathrm{min} / 1.73 \mathrm{~m}^{2}\right)\end{array}$ & $86.94 \pm 1.76$ & $79.56 \pm 3.40$ & $72.18 \pm 6.37$ & 0.025 & $86.67 \pm 1.76$ & $82.01 \pm 3.45$ & $77.34 \pm 6.49$ & 0.224 \\
\hline
\end{tabular}

${ }^{\mathrm{a}}$ GAT to GGT: major allele $=\mathrm{A}(96.4 \%)$ and minor allele $=\mathrm{G}(3.6 \%) ;{ }^{\mathrm{b}}$ AGC to AAC: major allele $=\mathrm{G}(96.5 \%)$ and minor allele $=\mathrm{A}(3.5 \%)$.

ipants who did not meet these criteria but reported to be under treatment with either oral antidiabetic agents or insulin and who gave a history of diabetes were also considered to have T2DM. GFR was estimated by the four-variable Modification of Diet in Renal Disease (MDRD) equation [12]. For the present study, we used the eGFR and TLR4 variant data available for the 434 participants.

Genotyping of Asp(299)Gly (rs4986790) and Thr(399)Ile (rs4986791) variants of TLR4 was performed using the TaqMan assay (Applied Biosystems, ABI, USA). Allelic discrimination PCR was carried out on a GeneAmp PCR system 9700 (ABI), and fluorescent signals were detected on an ABI PRISM 7700 sequence detector (ABI).

Statistical genetic association analysis was performed using the program SOLAR. Briefly, the genotypic data were checked for Mendelian consistency using the program SimWalk2 [13]. Allele frequencies were estimated using maximum likelihood techniques, which account for pedigree structure. The quantitative transmission disequilibrium test was carried out to examine hidden population stratification [14]. Association analysis was performed using the measured genotype approach within the variance components analytical framework implemented in SOLAR [15].

\section{Results}

Table 1 shows the clinical characteristics of the 434 genotyped individuals in this study. A total of $29 \%$ of the study participants (mean age $=46.9$ years, females $=61 \%$ ) had T2DM. The association results of the genotyped SNPs are presented in table 2 . The allele frequencies of A and G of the rs 4986790 variant of TLR4 were 96.4 and 3.6\%, respectively. With respect to rs4986791, the allele frequencies of $\mathrm{G}$ and $\mathrm{A}$ were 96.5 and 3.5\%, respectively. Genotypic data were in Hardy-Weinberg equilibrium. There was no evidence for hidden population stratification in the data as tested by the quantitative transmission disequilibrium test. Of the two SNPs in TLR4 examined for their association, the A to G substitution at codon 299 changing 
Thameem et al.: Genetic Variants in Toll-Like Receptor 4 Gene and Their Association

the amino acid from aspartic acid to glycine [Asp(299)Gly] with minor allele (G) frequencies of 3.6\% exhibited significant association with eGFR ( $\mathrm{p}=0.025$ ) after adjusting for the covariate effects of age, sex, body mass index, diabetes, duration of diabetes, systolic blood pressure, and use of antihypertensive medications. The model-derived mean eGFR values by genotype categories are shown in table 2. The carriers of the Gly299 variant had significantly decreased eGFR values. In addition, the $\mathrm{G}$ to A substitution at codon 399 changing the amino acid from threonine to isoleucine [Thr(399)Ile], with minor allele (A) frequencies of 3.5\%, failed to show an association with eGFR. The carriers of Ile399 indicated a trend towards a decrease in eGFR (table 2).

\section{Discussion}

Genome-wide linkage and association analyses have been employed to localize susceptibility genes influencing renal dysfunction $[16,17]$. However, genetic variants underlying this susceptibility have not been fully identified. In an effort to resolve this issue as a complementary approach, the present study investigated the influence of genetic variants of TLR4 on renal dysfunction and identified that the Asp(299)Gly variant in TLR4 is associated with a decrease in eGFR.

TLR4, a key member of the innate immune system, binds to the exogenous ligands, such as hyaluronan, heparan sulfate, or fibrinogen, and promotes renal injury by increasing inflammation [2]. Chronic inflammation-mediated activation of the innate immune system has been implicated in the pathogenesis of CKD/DN [2, 3]. In diabetes, TLR4 may sense high glucose, free fatty acids, and advanced glycation end products as signal molecules and trigger the inflammatory signaling cascade to contribute to DN [3]. In DN patients, TLR4 expression was found to be upregulated in glomeruli with a subsequent decline in eGFR [3]. TLR4 expression was associated with increased expression of TGF- $\beta 1$ in kidney biopsies from patients with CKD/DN $[3,18]$. In summary, it is evident that TLR4 activation likely contributes to DN.

Genetic variants in TLR4, particularly the Asp(299)Gly and Thr(399)Ile, have been associated with inflammatory diseases, including obesity, insulin resistance, T2DM, and the metabolic syndrome [5, 6]. However, the frequencies and role of these variants in regulating eGFR, a measure of renal function, have not been investigated in the Mexican American population. In this study, we observed a minor allele frequency of about 3.5\% for both the Gly299 and Ile399 variants in our Mexican American population, which is lower than those observed in Mexican and Caucasian populations. In the Mexican population, the minor allele frequencies of Gly299 and Ile399 were 5.9 and 6.3\%, respectively [19]. With respect to Caucasians, the minor allele frequencies of Gly299 and Ile399 were 7.9 and 11\%, respectively [20]. Ethnic differences in the occurrence of genetic variants at the TLR4 locus may relate to the ethnic specific predisposition to inflammation-mediated pathogenesis of disease.

of the two variants examined, this study observed a significant association between Asp(299)Gly and eGFR after accounting for the covariate effects. Interestingly, the carriers of Gly299 had significantly decreased eGFR. Although the functional significance of the association of the Gly299 variant of TLR4 with reduced renal function needs to be examined, the Gly299 variant of TLR4 may alter the ligand-binding capacity and its activation, balance between pro- and anti-inflammatory cytokines, and thereby may contribute to the chronic inflammation-mediated loss of renal function. In support of this statement, STZ-induced diabetic and uninephrectomized TLR4 ${ }^{-/-}$mice displayed a significant reduction in renal function, renal cortical NF- $\mathrm{KB}$ activation, tubular CCL-2 expression, and interstitial macrophage infiltration compared to wild-type animals [21]. 
Thameem et al.: Genetic Variants in Toll-Like Receptor 4 Gene and Their Association Analysis with Estimated Glomerular Filtration Rate in Mexican American Families

There are some limitations concerning the findings of this study. The GFR estimated by MDRD has not been validated in Mexican Americans. Our data are cross-sectional in nature and the eGFR was estimated using a single random blood sample for serum creatinine concentration. Therefore, a prospective study could be useful to precisely assess the effect of the Gly299 variant of TLR4 on the decline of renal function over time. Another major limitation of this study is the relatively small sample size. Given the sample size available for this study, there was $80 \%$ power to detect genetic influences that account for $2.2 \%$ of the phenotypic variation in eGFR at a significance level of 0.05 . However, the observed association with rs4986790 explained 1.04\% variation, in turn indicating the chances of finding this association was approximately one in two. Therefore, additional validation studies with increased sample sizes in ethnically diverse populations coupled with animal models could add additional strength to our current association finding. Apart from these, this study, for the first time, provides the evidence that the Asp(299)Gly variant in TLR4 associates with a decrease in eGFR in Mexican American participants of the SAFDGS, although it is a nominally statistically significant finding.

\section{Acknowledgements}

We thank the members of the SAFDGS for their participation and cooperation. This study was supported by the Grant-in-Aid award from the American Heart Association (F.T.), Carl W. Gottschalk Research Scholar Award from the American Society of Nephrology (F.T.), Norman S. Coplon Award (F.T.), and the Diabetes Action Research and Education Foundation (F.T.), George O’Brien Kidney Research Center (P50 DK061597), VA-Merit Review, and by grants from the NIDDK (DK42273, DK47482, and DK53889).

\section{Statement of Ethics}

The Institutional Review Board of the University of Texas Health Science Center at San Antonio approved all study procedures, and all subjects gave informed consent.

\section{Disclosure Statement}

None of the authors has competing interests related to this work.

\section{References}

1 Coresh J, Turin TC, Matsushita K, Sang Y, Ballew SH, Appel LJ: Decline in estimated glomerular filtration rate and subsequent risk of end-stage renal disease and mortality. JAMA 2014;311:2518-2531.

2 Lin M, Tang SC: Toll-like receptors: sensing and reacting to diabetic injury in the kidney. Nephrol Dial Transplant 2014;29:746-754.

3 Verzola D, Cappuccino L, D’Amato E Villaggio B, Gianiorio F, Mij M, et al: Enhanced glomerular Toll-like receptor 4 expression and signaling in patients with type 2 diabetic nephropathy and microalbuminuria. Kidney Int 2014;86:1229-1243.

4 Noreen M, Arshad M: Association of TLR1, TLR2, TLR4, TLR6, and TIRAP polymorphisms with disease susceptibility. Immunol Res 2015;62:234-252.

5 Weyrich P, Staiger H, Stančáková A, Machicao F, Machann J, Schick F, et al: The D299G/T399I Toll-like receptor 4 variant associates with body and liver fat: results from the TULIP and METSIM Studies. PLoS One 2010; 5:e13980.

6 Belforte FS, Coluccio Leskow F, Poskus E, Penas Steinhardt A: Toll-like receptor 4 D299G polymorphism in metabolic disorders: a meta-analysis. Mol Biol Rep 2013;40:3015-3020.

7 Ohto U, Yamakawa N, Akashi-Takamura S, Miyake K, Shimizu T: Structural analyses of human Toll-like receptor 4 polymorphisms D299G and T399I. J Biol Chem 2012;287:40611-40617. 
8 Prohinar P, Rallabhandi P, Weiss JP, Gioannini TL: Expression of functional D299G.T399I polymorphic variant of TLR4 depends more on coexpression of MD-2 than does wild-type TLR4. J Immunol 2010;184:4362-4367.

9 Park BS, Song DH, Kim HM, Choi BS, Lee H, Lee JO: The structural basis of lipopolysaccharide recognition by the TLR4-MD-2 complex. Nature 2009;458:1191-1195.

10 Figueroa L, Xiong Y, Song C, Piao W, Vogel SN, Medvedev AE: The Asp299Gly polymorphism alters TLR4 signaling by interfering with recruitment of MyD88 and TRIF. J Immunol 2012;188:4506-4515.

11 Puppala S, Dodd GD, Fowler S, Arya R, Schneider J, Farook VS, et al: A genomewide search finds major susceptibility loci for gallbladder disease on chromosome 1 in Mexican Americans. Am J Hum Genet 2006;78:377392.

12 Thameem F, Puppala S, Schneider J, Bhandari B, Arya R, Arar NH, et al: The Gly(972)Arg variant of Human Insulin Receptor Substrate 1 (IRS1) gene is associated with variation in Glomerular Filtration Rate (GFR) likely through impaired insulin receptor signaling. Diabetes 2012;61:2385-2393.

13 Sobel E, Lange K: Descent graphs in pedigree analysis: applications to haplotyping, location scores, and marker-sharing statistics. Am J Hum Genet 1996;58:1323-1337.

14 Abecasis GR, Cookson WO, Cardon LR: Pedigree tests of transmission disequilibrium. Eur J Hum Genet 2000; 8:545-551.

15 Almasy L, Blangero J: Exploring positional candidate genes: linkage conditional on measured genotype. Behav Genet 2004;34:173-177.

16 Thameem F, Igo RP Jr, Freedman BI, Langefeld C, Hanson RL, Schelling JR: A genome-wide search for linkage of estimated glomerular filtration rate (eGFR) in the Family Investigation of Nephropathy and Diabetes (FIND). PLoS One 2013;8:e81888.

17 Regele F, Jelencsics K, Shiffman D, Paré G, McQueen MJ, Mann JF, et al: Genome-wide studies to identify risk factors for kidney disease with a focus on patients with diabetes. Nephrol Dial Transplant 2015;suppl 4:iv26iv34.

18 Lepenies J, Eardley KS, Kienitz T, Hewison M, Ihl T, Stewart PM, et al: Renal TLR4 mRNA expression correlates with inflammatory marker MCP-1 and profibrotic molecule TGF- $\beta_{1}$ in patients with chronic kidney disease. Nephron Clin Pract 2011;119:c97-c104.

19 Maldonado-Bernal C, Trejo-de la OA, Sánchez-Contreras ME, Wacher-Rodarte N, Torres J, Cruz M: Low frequency of Toll-like receptors 2 and 4 gene polymorphisms in Mexican patients and their association with type 2 diabetes. Int J Immunogenet 2002;38:519-523.

20 Edfeldt K, Bennet AM, Eriksson P, Frostegård J, Wiman B, Hamsten A, et al: Association of hypo-responsive toll-like receptor 4 variants with risk of myocardial infarction. Eur Heart J 2004;25:1447-1453.

21 Tang SC, Lai KN: The pathogenic role of the renal proximal tubular cell in diabetic nephropathy. Nephrol Dial Transplant 2012;27:3049-3056. 